\title{
Antifouling Effect on Biomimetic Metamaterial Surfaces
}

\author{
Tomoki Nishino $^{1^{*}}$, Hiroshi Tanigawa $^{2}$, and Atsushi Sekiguchi ${ }^{3}$ \\ ${ }^{1}$ College of Science and Engineering, \\ ${ }^{2}$ The Research Organization of Science and Technology, \\ Ritsumeikan University, Kusatsu, Shiga 525-8577, Japan \\ ${ }^{3}$ Litho Tech Japan Corporation, \\ Kawaguchi, Saitama 332-0034, Japan \\ *rits0108@fc.ritsumei.ac.jp
}

\begin{abstract}
The snail shells have $200 \mathrm{~nm}$ porous structures, and an oil repellent function in water. Three biomimetic structures were fabricated: a silicon black surface, a silicon substrate having nano pillars, and a soft resin sheet having nano pillars. All these structures have equivalent sizes to the real snail shells, and showed good oil repellent effects. The latter two structures have regularly arranged pillars. The last resin sheet was fabricated by using a silicon mold with nano holes and using nanoimprint technologies. As this sheet is soft metamaterial, it can be processed to other shapes. As an example, a tube with oil repellent inner walls was fabricated and evaluated. Good oil repellent and antifouling effect were experimentally obtained from the water-oil mixture flow test. These results indicate the future advances in medical equipment and infrastructure.
\end{abstract}

Keywords: Biomimetics, Repel oil and water, Porous materials, Antifouling technology

\section{Introduction}

Living organisms are known to be efficient for survival in nature. Biomimetic technology, that mimics the organismic unique functions and shapes, involves a variety of fields, such as materials, machinery, therapy, environment, robotics and energy. A well-known example of biomimetic technology is the burdock. This finding led to the invention of Velcro ${ }^{\circledR}$. Recently, biomimetic technology has been actively conducted. In particular, a great deal of research has focused on the organismic surface structure. For example, morpho butterflies fine structures were reproduced on $\mathrm{Si}$ substrates [1] for super-hydrophobicity, the study of cherries with attachment and detachment functions [2], dragonfly wings showed physically killing bacteria [3], and abalone crust showed its strong structure, hardly destroyed even at the same thickness [4].

These studies on the microscopically projected structure have implications for new possibilities.

In this paper, biomimetic technologies with oil repellent and antifouling effects are focused. It is known that the surface structure of the snail shell has an oil repellent effect by superhydrophilicity [5-10]. Semiconductor technologies, are applied to mimic the snail shells structure for demonstrating the oil repellent effect.

\section{Oil-repellent in material with a shape similar to snail shells}

The surface of snail shells was observed by using SEM. As shown in Fig. 1, the surface structure is composed of a concave area (200-400 $\mathrm{nm})$ and a convex area (200-400 nm). It has been

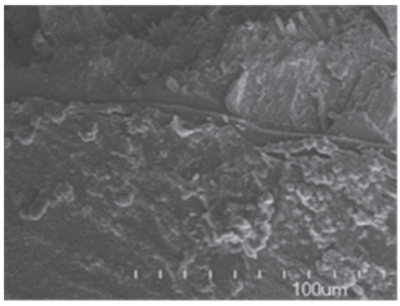

(a)

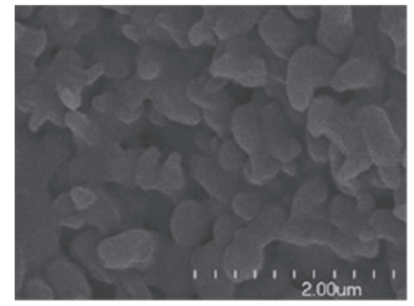

(b)
Fig. 1. Snail shells SEM photos: (a) whole shells, and (b) enlarged shells. 


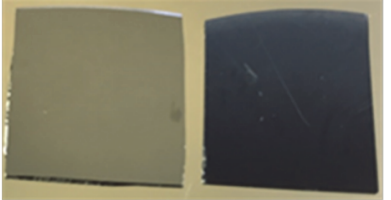

(a)

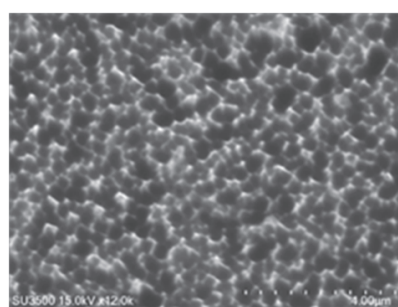

(b)
Fig. 2. Black silicon SEM photos: (a) Si substrate, and (b) enlarged black silicon.

shown that snail shells have an oil repellent function in water. Therefore, it is very interesting whether a counterfeit structure of a snail shell exhibits a similar oil repellent function

The first mimic, black silicon on a silicon wafer, was fabricated by dry etching. As shown in Fig. 2, the black silicon surface has $200 \mathrm{~nm}$ unevenness.

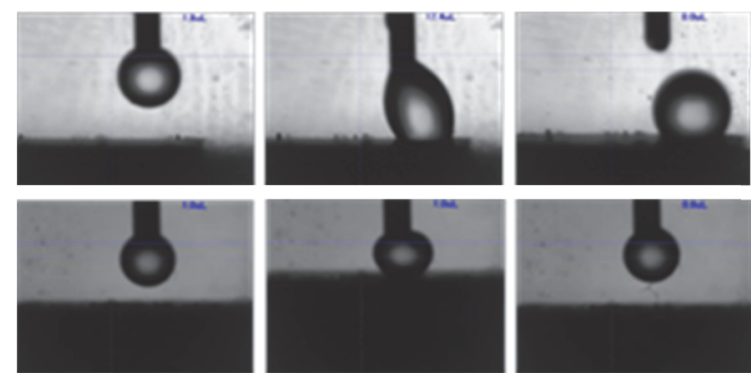

Fig. 3. Oil repellent in water: (a) no repellent on Si bare substrate, and (b) repellent on black silicon.

Figure 3 shows the oil repellent effect in water, for the Si bare substrate (mirror surface) and for the black silicon (no reflection). When the oil droplets are brought into contact with the Si bare substrate, they adhere to the substrate. On the other hand, when the oil droplets are brought into contact with the black silicon surface, they do not adhere to the surface. Thus, it was clearly found that the same $200 \mathrm{~nm}$ of structures as the snail shell have oil repellent functions.

\section{Oil repellent on silicon pillar surface}

As shown in Fig. 3, black silicon surface, with randomly distributed unevenness, is hydrophilic. To analyze the hydrophilicity, regularly arranged uneven surfaces with holes and pillars are attractive.

Figures 4(a) and (b) show Si substrate having nano pillars on the surface. The starting material is a Si substrate. Nano pillars, with $200 \mathrm{~nm}$ height and $200 \mathrm{~nm}$ diameter in equal spacing, were fabricated by EB-exposure and deep etching (RIE, reactive ion etching).
The oil repellent on the Si nano pillar surface is shown in Fig. 4(c). Good oil repellent is demonstrated; equivalent to that for silicon black surfaces.

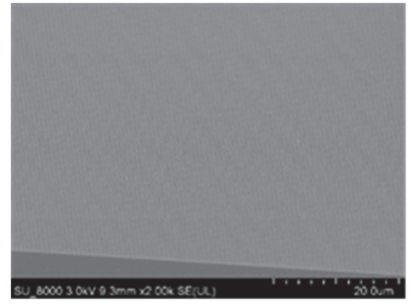

(a)

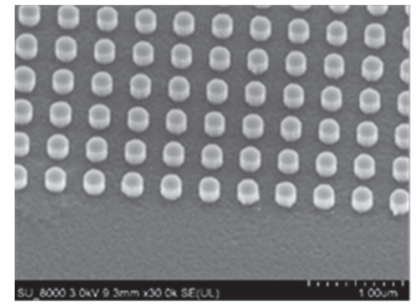

(b)

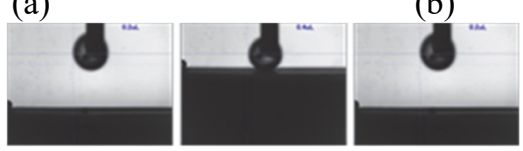

(c)

Fig. 4. Silicon nano pillars: (a) SEM photo $(\times 2,000)$, (b) SEM photo $(\times 30,000)$, and $(c)$ oil repellent in water.

\section{Oil repellent sheet}

Though a silicon substrate is attractive for the nano-size fabrication, it is not suited to a mass production with lower cost. Moreover, if an oil repellent material is soft and easy to warp, it can be applied by processing to such complex shapes as tubes and curved surfaces. For these reasons, an oil repellent "soft sheet" was fabricated by combining a mold having nano holes and nano imprinting.

The starting mold material is a Si substrate. Nano holes, with $200 \mathrm{~nm}$ depth and $200 \mathrm{~nm}$ diameter in equal spacing, were fabricated on its surface, by following the above described processes. Figure 5 shows the fabricated silicon mold.

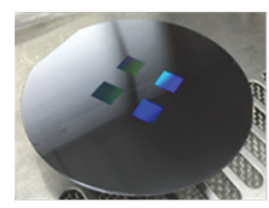

(a)

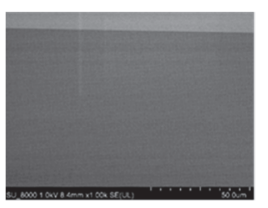

(b)

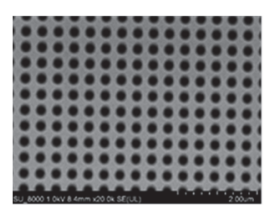

(c)
Fig. 5. Silicon substrate with nano pillars: (a) Si wafer, (b) SEM photo $(\times 1,000)$, and (c) SEM photo $(\times 20,000)$.

Next, a UV curable acrylic resin [11] is dropped onto the mold and spun to a thin layer. The Si mold is pressed to the resin layer, and the resin is cured by UV irradiation. The nanoimprint machine LTNIP-5000 [12] (Lithotec Japan Co., Ltd.) was used. In nanoimprint process, the pressing pressure was $1000 \mathrm{~N}$, the UV-light $(365 \mathrm{~nm} \sim 436 \mathrm{~nm})$ intensity was $30 \mathrm{~mW} / \mathrm{cm}^{2}$, and the exposure time was 5 minutes. After curing the resin with UV irradiation, the Si mold was released.

The fabricated resin sheet, having biomimetic 
structure, is shown in Figs. 6(a) -(c). Figure 6(d) shows the oil repellent effect on the resin sheet with nano pillars. As the resin sheet with no pillars has no oil repellent function, the nano pillar processed resin sheet, having an oil repellent, is a class of metamaterials, engineered to produce properties that don't occur naturally.

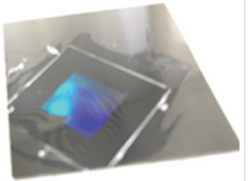

(a)

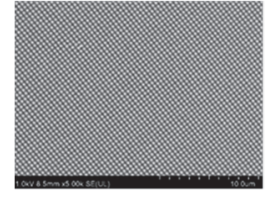

(b) (c)
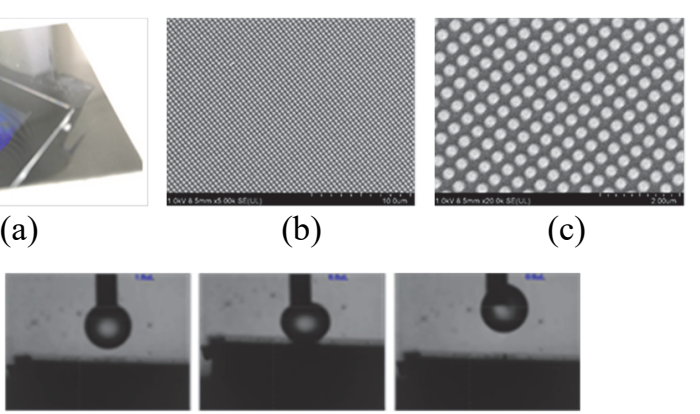

(d)

Fig. 6. Fabricated nanoimprinted resin sheet with nano pillars: (a) imprinted sheet, (b) SEM photo $(\times 5,000)$, (c) SEM photo $(\times 20,000)$, and (d) Oil repellent in water.

\section{Oil repellent tube}

For an example of feasible practical applications, the fabricated soft resin sheet was processed to a tube, whose inner wall would be oil repellent. The test tube consists of a left part (Fig. 7(a)) with no biomimetic structure, and a right part with biomimetic structure (having nano pillars).

The tube is set to the test equipment as shown in Fig. 8; the fluid flows through the tube at a constant rate, and the tube inner wall is monitored by a video camera.

In the experiment, the fluid was mixed with 9 (water): 1(pork lard, red colored for observation) by weight. The fluid temperature was 40 degrees centigrade. The fluid flow into the tube intermittently; water (with no oil) - oil mixture water (with no oil).

Figure 7 shows the oil repellency effect. Once the oil mixture flows, the inner wall for the non-biomimetic part becomes dirty and soil, even after water flows (Fig. 7(c)). On the other hand, the other inner wall with biomimetic structure is clean and antifouling.

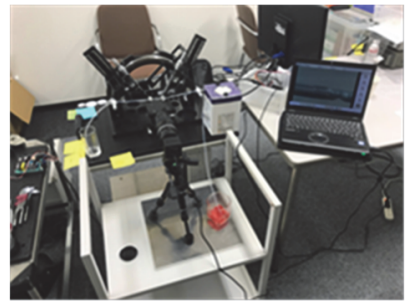

(a)

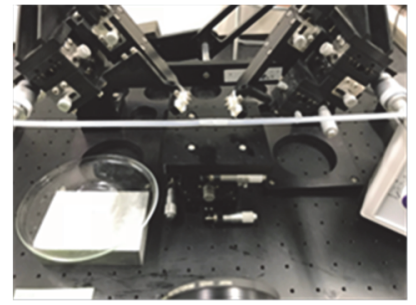

(b)
Fig. 8. Liquid flow test equipment: (a) setup, and (b) water and oil flow in the tube.

\section{Conclusion}

Oil repellent and antifouling biomimetic structures were fabricated and evaluated. Silicon black surface, silicon substrate with nano pillars, and nanoimprinted resin sheet with nano pillars are good oil repellent in water. These structures have unevenness, corresponding to the real snail shells (around $200 \mathrm{~nm}$ ). Especially the nanoimprinted soft resin sheet, a class of metamaterials, has processing merits to fabricate complex surfaces. As an example, the slender tube was fabricated and evaluated the oil repellent. These experimentally obtained results would be contributed to advance medical equipment and infrastructure technologies.

\section{Acknowledgements}

The authors would like to express their deepest gratitude to Mr. Y. Inoue of Kyoto University's Nano Hub Base for the provision of research equipment and proposals for this research. This research was supported by the New Technology Products Realization and Development Grants Project of Saitama Prefecture for FY2017.

\section{References}

1. K. Watanabe, T. Hoshino, K. Kanda, Y. Haruyama, and S. Matsui, Jpn. J. Appl. Phys., 44 (2005) L48.

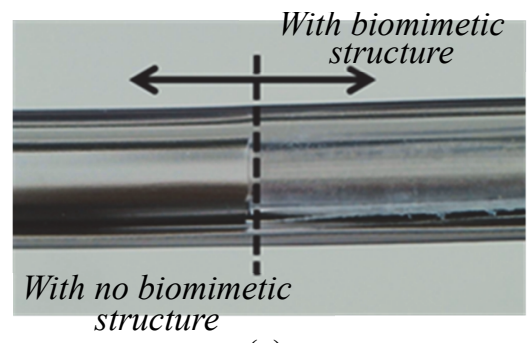

(a)

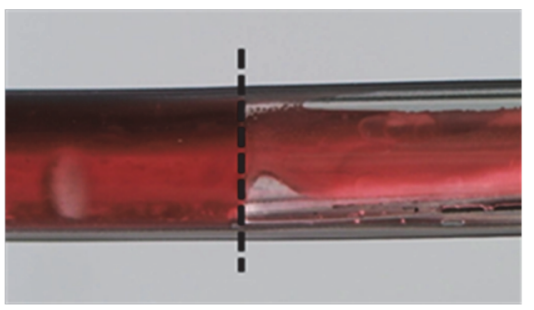

(b)

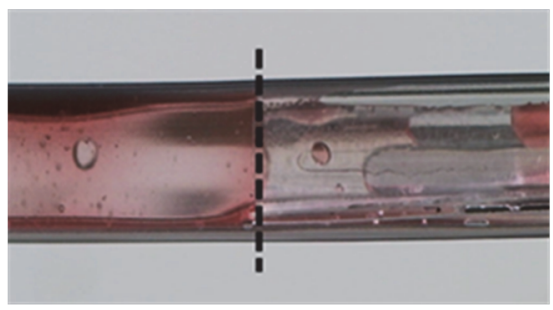

(c)

Fig. 7. Oil repellent for the fabricated tube: (a) before flowing liquid, (b) while flowing water-oil mixture, and (c) after flowing water-oil mixture. 
2. K. Autumn, Y. A. Liang, S. T. Hsieh, W. Zesch, W. P. Chan, T. W. Kenny, R. Fearing, and R. J. Full, Nature, 405 (2000) 681.

3. E. P. Ivanova, J. Hasan, H. K. Webb, V. K. Truong, G. S. Watoson, J. A. Watson, V. A. Baulin, S. Pogodin, J. Y. Wang, M. J. Tobin, C. Lobbe, and R. J. Crawford, Small, 16 (2012) 2489.

4. A. Finnemore, P. Cunha, T. Shean, S. Vignolini, S. Guldin, M. Oyen, and U. Steiner, Nat. Commun., 3 (2012) 966.

5. A. G. Domel, M. Saadat, J. C. Weaver, H. H. Hariri, K. Bertolodi, and G. V. Lauder, J. R. Soc. Interface, 15 (2018) 1742.

6. M. Shimomura, "Biomimetics" Tokai university Pub. Co., Tokai (2016) 16.

7. J. Knippers, K. G. Nickel, and T. Speck,
"Biomimetic Research for Architecture and Building Construction" Springer International Pub. Co., Switzerland (2016) 408.

8. R. Nishimura, K. Hyodo, H. Sawaguchi, Y. Yamamoto, Y. Nonomura, H. mayama, S. Yokojima, S. Nakamura, and K. Uchida, J. Am. Chem. Soc., 138 (2016) 10299.

9. K. Takase, K. Hyodo, M. Morimoto, Y. Kojima, H. Mayama, S. Yokojima, S. Nakamura, and K. Uchida, Chem. Commun., 52 (2016) 6885.

10. L. Ren, J. Tong, J. Li, and B. Chen, J. Agric. Engng. Res., 79 (2001) 239.

11. N. Sakai and H. Hirasawa, Proc. Soc. Polym. Sci., Jpn., 66 (2009) 88.

12. A. Sekiguchi, Y. Kono, and Y. Hirai, $J$. Photopolym. Sci. Technol., 18 (2005) 543. 